This manuscript is a non-peer reviewed preprint submission to the EarthArxiv. It has not been accepted for formal publication. Subsequent versions of this manuscript may contain slightly different content. Please feel free to contact the author. 


\title{
Forecasting the localized bilateral effects of ocean acidification on the counter carbonate pump using recurrent neural networks
}

\author{
Eshan Ramesh ${ }^{1}$ \\ ${ }^{1}$ Oviedo High School, esrh@netc.eu
}

\begin{abstract}
The counter carbonate $\operatorname{pump}(\mathrm{CCP})$ is responsible for carbon dioxide sequestration and cycling forms of carbon in the ocean. It is primarily driven by calcifying plankton, such as foraminifera, coccolithophores, and pteropods. These organisms are particularly vulnerable to ocean acidification, which can have disastrous effects on their skeletons and productivity, upsetting the marine carbon cycle in ways that have not been quantified due to their chaotic nature. This project aims to provides highresolution, accurate, and robust predictions of the efficiency of the CCP. These predictions are achieved by training recurrent neural networks on satellite-determined particulate inorganic carbon(PIC) to particulate organic carbon(POC) ratio data that represents the efficency of calcifying organisms in exporting PIC. Recurrent neural networks mimic the way in which biological neurons learn patterns and recall past experiences, and have been shown to be good at predicting chaotic time series. For each $9 \times 9 \mathrm{~km}$ square sample in a random subset of the dataset, the time series of historical PIC, POC, tropospheric satellite $\mathrm{CO} 2$ data, latitude and longitude were used by the model. The applications of the model are threefold. First, it can pinpoint the most vulnerable area in the ocean at arbitrary times in the future. Second, general trends in PIC are a proxy for ocean acidification. Finally, anomalous spikes in PIC can be potential coccolithophore blooms, which are dangerous for sub-surface marine life. The model predicts the ratio value to an error of $1.3 \%$, tested using cross validation, and is significantly better than linear regression. Future directions for work include physically testing affected specimens in projected water characteristics.
\end{abstract}

\section{INTRODUCTION}

Calcifying organisms are some of the most vulnerable to ocean acidification(OA) caused by rapidly increasing anthropogenic carbon in the oceans. Plankton such as coccolithophores are driving forces in the ocean surface carbon cycle, but are sensitive to subtle changes of acidity [1]. However, the patterns of where and when these calcifying organisms are affected are unknown, thus this project aims to provide hyperlocalized, accurate, extensible, and robust predictive power across the globe. The ratio between particulate inorganic carbon(PIC) and particulate organic carbon(POC) describes the efficiency of the counter carbonate pump, which is responsible for the removal of $\mathrm{CO} 2$ from the ocean as well as the export of calcium carbonate [2]. These two carbon values are determinable from satellite backscatter data, using an existing algorithm developed from and validated on field measurements [3]. This provides a reliable and large source of historical data. Recurrent neural networks have been shown to be extraordinarily good at learning chaotic time series patterns
[4]. Thus, it is hypothesized that they will be good fits for predicting ocean acidification.

This model could be used to pinpoint the most vulnerable area of ocean at any point in time. Trends in the PIC:POC ratio are also indirectly correlated to $\mathrm{pH}$, so this also tracks relative changes in ocean acidification using particulate carbon as a proxy. Furthermore, anomalous spikes in the ratio can potentially indicate phytoplankton blooms, which are dangerous to subsurface marine flora and fauna. The ability to pinpoint future changes in acidification and its effects will allow scientists to prepare for and mitigate some of these problems.

\section{A. Anthropogenic carbon dioxide and its effects on the ocean}

Carbon dioxide levels have been rising exponentially since the dawn of the Industrial Revolution; humans have added about 400 billion tons to the atmosphere [5]. Naturally, the ocean absorbs $\mathrm{CO}_{2}$ through the dissolution, becoming more acidic in a process known as ocean acidification(OA).

Ocean acidification is occurring faster than ever before in geologic history and is currently of critical importance [6]. It has severe impacts on marine life. Calcifying organisms, like corals, that build shells or exoskeletons out of $\mathrm{CaCO}_{2}$ are of exceptional significance because calcium carbonate dissolves in acidic water [7], [8]. Surface calcifying plankton like coccolithophores, foraminefera, and pteropods, which are responsible for sequestering $\mathrm{CO}_{2}$ back into the atmosphere are especially vulnerable. This has led to a positive feedback loop in which the primary organisms affected by OA are the ones that typically mitigate it [2], [7]. Also affected are organisms like oysters and other shellfish, which are important economically to global fisheries. It is estimated that by 2100 , ocean acidification may cost over 100 billion dollars [9].

\section{B. Overview of relevant ocean carbon chemistry}

The oceans have incredibly complex element cycles. With respect to carbon, there are 4 aquatic forms that are distinguished by their state and source. Particulate inorganic carbon(PIC) and particulate organic carbon(POC) are both suspended carbon matter. PIC is primarily produced by calcifying surface organisms, and is mostly calcium carbonate. POC is produced by living organisms photosynthesizing, and is found in organic detritus. 
The other two forms of carbon are dissolved in the water, and are either inorganic, or organic. Dissolved inorganic carbon(DIC) is formed when $\mathrm{CO}_{2}$ dissolves in the ocean. It forms carbonic acid, $\mathrm{HCO}_{3}$, which then disassociates into the intermediary bicarbonate ions, $\mathrm{HCO}_{3}{ }^{1-}$, and further disassociates into carbonate ions, $\mathrm{CO}_{3}{ }^{2-}[10]$. Of particular interest is the ratio between POC and PIC, also known as the "rain ratio", because it represents the strength of the counter carbonate pump. This is the process by which calcifying plankton build their calcium carbonate shells and release $\mathrm{CO}_{2}$ as a byproduct [11].

\section{Historical studies}

The PIC:POC ratio has been well studied with respect to plankton. In correlational studies, the ratio has been shown to correlate with $\mathrm{pH}$ and total alkalinity, both values that are directly related to ocean acidification [12]. Other researchers have shown the ratio to be a good indicator of coccolithophore sensitivities to ocean acidification [13]. This means that tracking the PIC:POC value is by proxy tracking ocean acidification in general through the counter-carbonate pump. It is important to note that PIC:POC is simply an indicator, and does not directly cause any effects in the ocean.

Forecasting OA using computational models is not a new idea. [14] attempted to forecast ocean acidification in the Northwest Mediterranean Sea using a cubic regression based on field-acquired data points. [15] repeated a similar strategy in the Southern Ocean, collecting data along the path of a scientific ship. Current work in forecasting OA has three flaws: it is spatiotemporally specific, it uses outdated and simple modeling techniques, and is not time-extensible. This project aims to fill that gap using a novel strategy: forecasting the satellite-determined PIC:POC ratio using recurrent neural networks.

\section{Overview of neural networks}

Neural networks are a relatively novel strategy for computational modeling. A neural network imitates the way biological brains learn patterns from experience. A simple sequential model is composed of many layers of nodes known as "neurons". In a classic neural network called a "multi layer perceptron", each of the neurons in each layer is connected to all of the neurons in the adjacent layers via "edges" [16].

In Figure 11, the model is designed to accept 2 values as inputs, named $x_{i}$. During the training phase of the model, during which the model actually learns, data is fed into the model beginning at the first layer. For every datapoint, each value is passed through the interconnecting edges and multiplied by a weight $w_{i}$. Once a value reaches a node, it is summed with all the other values coming from the edges in the previous layer. This sum is evaluated against a threshold called a bias. Learning occurs by adjusting these weight and biases.

Using mutlilayer perceptrons for time series is a suboptimal method, because they are limited to making one-step forward predictions. Recurrent neural networks(RNNs) are a newer type of neural network that avoids this flaw. In a recurrent

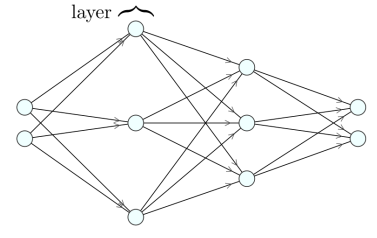

Fig. 1: Example multi-layer perceptron.

neural network, a time series can be analyzed by using multiple historical datapoints, i.e. using many more inputs than an MLP. In a long-short-term-memory layer, the output of a node is reused as input for the next sample. This makes it a good fit for the problem of forecasting the PIC:POC ratio globally, which is a time series.

\section{Methodology}

\section{A. Data preparation}

$\mathrm{CO}_{2}$, along with previous PIC, POC, latitude and longitude values were used as inputs for the model. They were acquired from open-source NASA datasets [17] [18] [19] The model was then trained to predict the next two PIC and POC values using these inputs.

3 sets of consequent $\mathrm{CO}_{2}$, PIC, POC, Latitude and longitude values were paired with the next PIC and POC values in that sequence. This results in a 3-dimensional dataset that is necessary to be used in a recurrent neural network. Each variable, $X_{i}$ was scaled around its mean such that $\overline{X_{i}}=0$ and $\sigma\left(X_{i}\right)=1$. This is done to speed up model training and is a standard procedure in data science [20].

\section{B. Neural network}

A stacked neural network architecture was used. Two 1000neuron long-short-term-memory(LSTM) networks were constructed, and a normal fully-connected layer was added as an output layer, containing 2 neurons. These two neurons represent the predicted PIC and POC values. This structure is shown graphically in figure 2 Another approach was also tested, except with a one-neuron exit layer, in which the model was trained to predict the PIC to POC ratio directly. This structure was determined from a systematic search through neural network architectures.

The models were trained using the ADAM(adaptive moment estimation) optimizer algorithm, which is computationally efficient and suitable for problems with large amounts of data [21]. Due to the complexity of the models and size of the dataset, training was done on an Nvidia K80 Graphics Processing Unit because of accessibility.

In order to robustly validate the models, 5-fold cross validation(CV) was used. The dataset was divided into five equal parts, and the model was repeatedly trained on four parts 


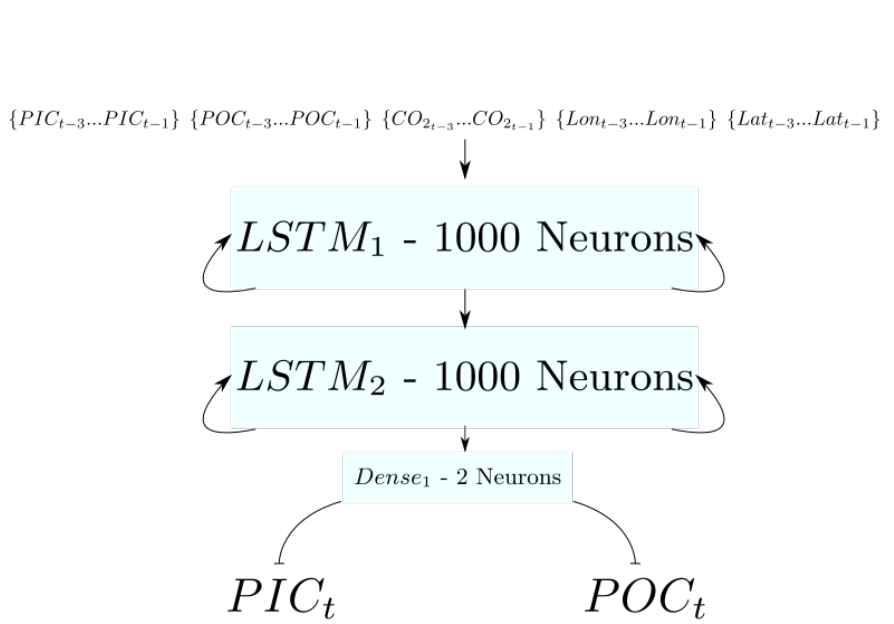

Fig. 2: Recurrent neural network structure used. Very complex with millions of trainable parameters to capture complex patterns.

and tested on the last part. The performance, measured in percent error, on each of the five trainings was averaged. After the process, the model would have been tested on the full dataset without being trained on it, so it is a balanced metric between bias and variance. 5-fold CV was used over other techniques like Leave-One-Out $\mathrm{CV}$ and 10 -fold $\mathrm{CV}$ because it is computationally more efficient and has been shown to perform almost as well as the other strategies [22].

\section{RESULTS}

The PIC and POC model achieved an error percentage of $1.3 \%$, which is significantly better than simple linear regression techniques and the direct ratio model by a $t$ distribution confidence interval, as shown in figure 3 . It is important to note that the model's individual PIC and POC errors, shown in figure 4. were only slightly increased from the overall ratio errors, achieving an error of $1.6 \%$ PIC error, and $-0.8 \%$ POC error. Using this neural network, it is possible to generate geographical maps with forecasted PIC/POC values. A sample of this for the coasts of Florida is shown. The high export ratio values near the northern east coast of Florida may be due to increased human marine traffic at and surrounding Jacksonville.

\section{DISCUSSION}

Because the PIC\&POC model's error falls far below the lowest $95 \%$ confidence error bound of the other two models, it is concluded that the model is significantly better. The results are encouraging, because it shows that neural networks can effectively capture extremely complex and chaotic natural patterns. It is very important to note however, that this low error is likely due in part to the scale of data measured. The majority of the ocean does tend to have inevitably lower carbonate values than some outlier regions where the model's predictions are most useful.
Average error percentage of ratio predictions by model

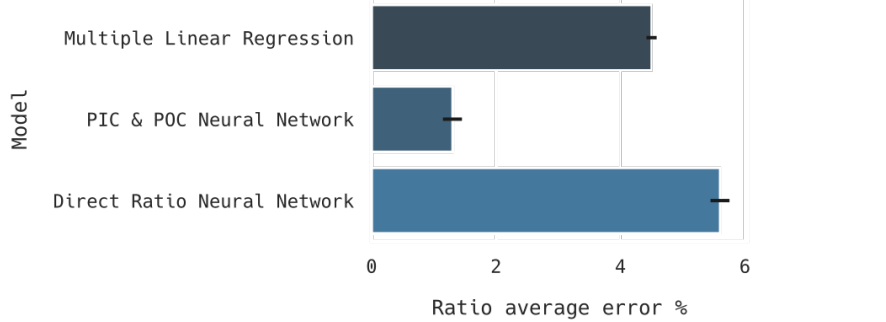

Fig. 3: Error percentages of models. 95\% $t$ confidence interval is plotted. Note that the PIC and POC model's error is lower than the bottom bounds of the intervals of both of the models.

\section{PIC and POC model error percentages}

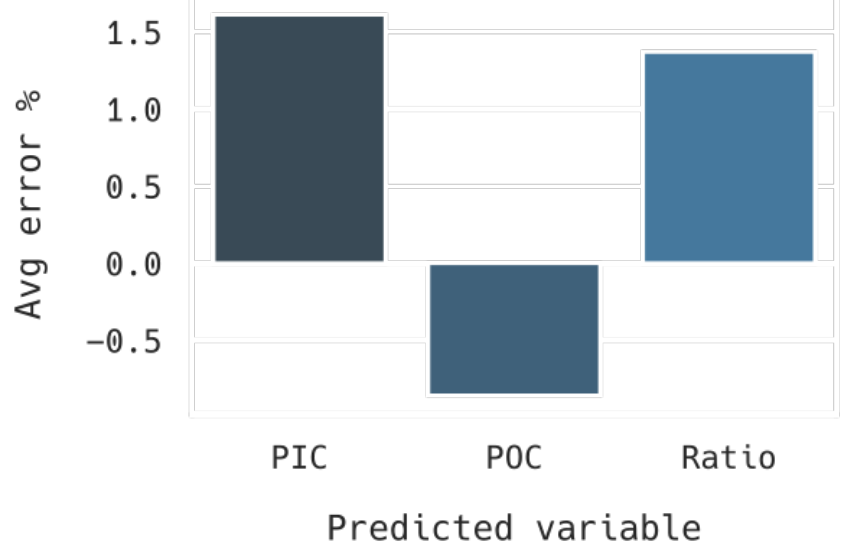

Fig. 4: Breakdown of PIC-POC model errors by individual variable. POC error was individually predicted to an absolute error less than that of the forecasted ratio.

Scientists are already developing several techniques to directly mitigate OA's effects, such as iron fertilization, which involves the artifical distribution of $\mathrm{FeSO}_{4}$ into the water [11]. This project may be able to provide the short-term data necessary to make practical decisions such as where and when to use iron fertilization. It is also currently critical to begin considering long-term action, which is best supported by data predictions. The presented neural network could also meet this demand as well, suggesting to governments or supranational organizations the optimal area to distribute funds in order to have the greatest impact. As mentioned earlier, predicting PIC and POC individually offers invaluable insight into several ocean processes. High PIC values, such as those near busy ports, imply high calcifying plankton activity. This kind of activity often results in blooms that deprive sub-surface life of necessary nutrients.

Neural networks challenging to design because they are difficult to interpret and very complex. They require fine tuning hundreds of parameters including architecture and compile options. Although great effort was taken to methodically and systematically search through the possible model space, it is likely that several better models were never realized. One possible method to improve performance would be to include 


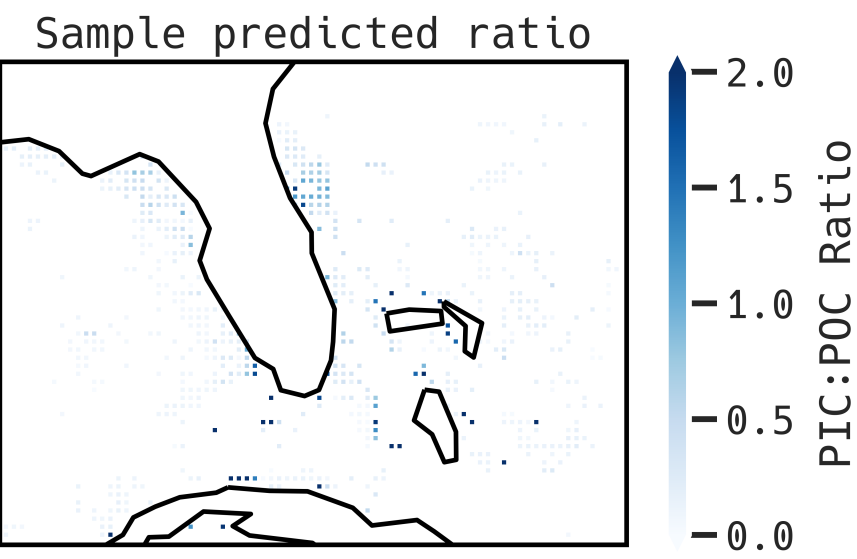

Fig. 5: Sample predicted ratio values in Florida, for the 8-day period beginning on January 1st 2017

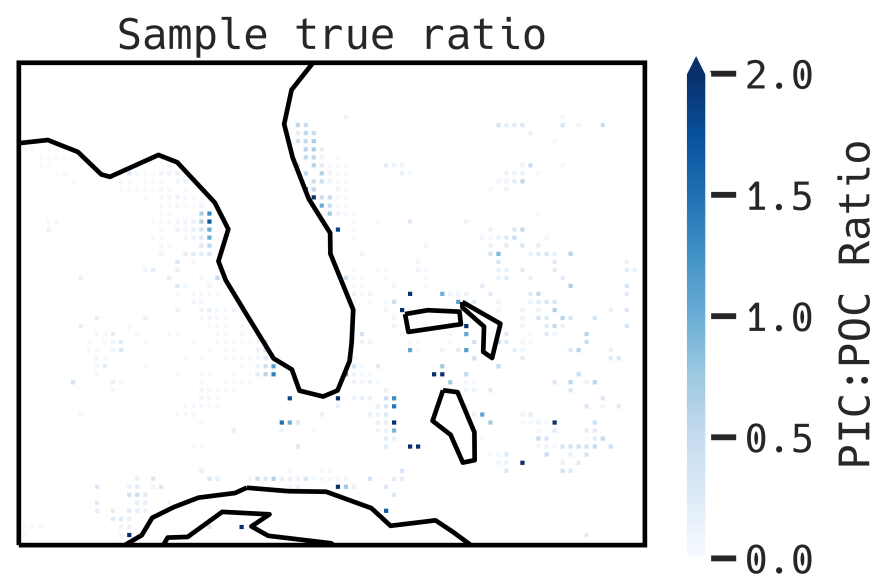

Fig. 6: Sample real ratio values in Florida, for the 8-day period beginning on January 1st 2017

a dropout layer in the model to combat overfitting by randomly dropping neural connections. This is the most significant source of error and warrants a more thorough investigation. As with any computational model, it is critical to verify that predictions were reasonable and accurate with the real world. This could be done through acquiring more field data regarding the PIC to POC ratio and comparing it to forecasted values.

\section{CONCLUSION}

Neural networks were hypothesized to be exceptionally good at forecasting PIC and POC because they fit chaotic natural time series well. The results confirm this hypothesis and suggests that these models may be applied to several other fields as well.

There are several directions for future work on this concept. Firstly, it is mathematically guaranteed that there is a better neural network model according to the Universal Approximation Theorem. Therefore, there are certainly ways to improve the accuracy and robustness of the model, by training it on more data and making the model more complex by increasing hidden layers and neuron counts. It should be noted that computational resource is a severely limiting factor is increasing model performance, as training more complex models requires exponentially more power. Another possible avenue of inquiry is to explore the real effects of projected acidity conditions on physical coccolithophore samples. This would be extraordinarily profound in that it would provide extremely good forecasts of the actual effects of ocean acidification on these species available.

Artificial intelligence is changing the way humans understand the complex patterns of the world. This model helps capture one of those patterns: ocean acidification and its effects. Because of its scalability and robustness, it provided both practical and long-term insights that could be instrumental in delivering the much-needed information to combat ocean acidification more effectively.

\section{ACKNOWLEDGMENTS}

The author thanks Mr. William Furiosi (Oviedo High School) for guidance throughout the project and paper's development, the National Aeronautics and Space Administration for their open-access data which made this project possible, and the contributors to all the free/open-source projects that were used in this project: Tensorflow, Python, NumPy, MatPlotLib, Xarray, NetCDF4, SciPy, and SciKit-Learn.

\section{REFERENCES}

[1] L. Beaufort, I. Probert, T. d. Garidel-Thoron, E. M. Bendif, D. Ruiz-Pino, N. Metzl, C. Goyet, N. Buchet, P. Coupel, M. Grelaud, B. Rost, R. E. M. Rickaby, and C. d. Vargas, "Sensitivity of coccolithophores to carbonate chemistry and ocean acidification," Nature, vol. 476, pp. 80-83, Aug. 2011, ISSN: 1476-4687. DOI: 10. 1038/ nature10295. [Online]. Available: https : // Www . nature . com / articles / nature 10295 (visited on 10/03/2019).

[2] C. Manno, F. Giglio, G. Stowasser, S. Fielding, P. Enderlein, and G. A. Tarling, "Threatened species drive the strength of the carbonate pump in the northern scotia sea," Nature Communications, vol. 9, no. 1, pp. 1-7, Nov. 2, 2018, ISSN: 2041-1723. DOI: 10.1038/s41467018 - 07088 - y. [Online]. Available: https : / / www . nature.com/articles/s41467-018-07088-y (visited on 01/08/2020).

[3] W. M. Balch, H. R. Gordon, B. C. Bowler, D. T. Drapeau, and E. S. Booth, "Calcium carbonate measurements in the surface global ocean based on moderateresolution imaging spectroradiometer data," Journal of Geophysical Research: Oceans, vol. 110, C7 2005, ISSN: 2156-2202. DOI: 10.1029/2004JC002560. [Online]. Available: https ://agupubs . onlinelibrary. wiley. com / doi / abs / 10 . 1029 / 2004JC002560 (visited on 09/17/2019).

[4] M. Han, J. Xi, S. Xu, and F.-L. Yin, "Prediction of chaotic time series based on the recurrent predictor neural network," IEEE Transactions on Signal Processing, vol. 52, no. 12, pp. 3409-3416, Dec. 2004, ISSN: 19410476. DOI: $10.1109 /$ TSP.2004.837418 
[5] A. Borunda. (Jun. 7, 2019). "Ocean acidification, explained," National Geographic, [Online]. Available: https : / / www . nationalgeographic . com / environment / oceans/critical-issues - ocean- acidification//visited on 09/11/2019).

[6] B. Hönisch, A. Ridgwell, D. N. Schmidt, E. Thomas, S. J. Gibbs, A. Sluijs, R. Zeebe, L. Kump, R. C. Martindale, S. E. Greene, W. Kiessling, J. Ries, J. C. Zachos, D. L. Royer, S. Barker, T. M. Marchitto, R. Moyer, C. Pelejero, P. Ziveri, G. L. Foster, and B. Williams, "The geological record of ocean acidification," Science, vol. 335, no. 6072, pp. 1058-1063, Mar. 2, 2012, ISSN: 0036-8075, 1095-9203. DOI: 10.1126/science.1208277. [Online]. Available: https://science. sciencemag.org/ content/335/6072/1058 (visited on 09/27/2019).

[7] A. J. Andersson, D. I. Kline, P. J. Edmunds, S. D. Archer, N. Bednaršek, R. C. Carpenter, M. Chadsey, P. Goldstein, A. G. Grottoli, T. P. Hurst, A. L. King, J. E. Kübler, I. B. Kuffner, K. R. Mackey, B. A. Menge, A. Paytan, U. Riebesell, A. Schnetzer, M. E. Warner, and R. C. Zimmerman, "Understanding ocean acidification impacts on organismal to ecological scales," Oceanography, vol. 28, no. 2, pp. 16-27, 2015, ISSN: 1042-8275. [Online]. Available: https://www.jstor.org/ stable/24861866 (visited on 10/03/2019).

[8] R. Albright, L. Caldeira, J. Hosfelt, L. Kwiatkowski, J. K. Maclaren, B. M. Mason, Y. Nebuchina, A. Ninokawa, J. Pongratz, K. L. Ricke, T. Rivlin, K. Schneider, M. Sesboüé, K. Shamberger, J. Silverman, K. Wolfe, K. Zhu, and K. Caldeira, "Reversal of ocean acidification enhances net coral reef calcification," $\mathrm{Na}$ ture, vol. 531, no. 7594, pp. 362-365, Mar. 2016, ISSN: 1476-4687. DOI: $10.1038 /$ nature17155. [Online]. Available: https://www.nature.com/articles/nature17155 (visited on 09/11/2019).

[9] D. Narita, K. Rehdanz, and R. S. J. Tol, "Economic costs of ocean acidification: A look into the impacts on global shellfish production," Climatic Change, vol. 113, no. 3, pp. 1049-1063, Aug. 1, 2012, ISSN: 1573-1480. DOI: 10.1007/s10584-011-0383-3. [Online]. Available: https://doi.org/10.1007/s10584-011-0383-3 (visited on $02 / 18 / 2020)$.

[10] S. Emerson and J. Hedges. (Apr. 2008). "Chemical oceanography and the marine carbon cycle," Cambridge Core, [Online]. Available: /core / books / chemical oceanography - and - the - marine - carbon - cycle / EA962F7A4363031C2CB89036CCEE65BE (visited on 01/15/2020).

[11] I. Salter, R. Schiebel, P. Ziveri, A. Movellan, R. Lampitt, and G. A. Wolff, "Carbonate counter pump stimulated by natural iron fertilization in the polar frontal zone," Nature Geoscience, vol. 7, no. 12, pp. 885-889, Dec. 2014, ISSN: 1752-0908. DOI: 10 . 1038/ngeo2285. [Online]. Available: https ://www . nature.com/articles/ngeo2285 (visited on 02/18/2020).

[12] H. S. Findlay, P. Calosi, and K. Crawfurd, "Determinants of the PIC : POC response in the coccolithophore emiliania huxleyi under future ocean acidification sce- narios," Limnology and Oceanography, vol. 56, no. 3, pp. 1168-1178, 2011, ISSN: 1939-5590. DOI: 10.4319/ lo.2011.56.3.1168. [Online]. Available: https://aslopubs. onlinelibrary.wiley.com/doi/abs/10.4319/lo.2011.56.3. 1168 (visited on 01/07/2020).

[13] N. A. Gafar, B. D. Eyre, and K. G. Schulz, "Particulate inorganic to organic carbon production as a predictor for coccolithophorid sensitivity to ongoing ocean acidification," Limnology and Oceanography Letters, vol. 4, no. 3, pp. 62-70, 2019, ISSN: 2378-2242. DOI: 10 . 1002/lol2.10105. [Online]. Available: https://aslopubs. onlinelibrary. wiley.com/doi/abs/10.1002/lol2.10105 (visited on 09/19/2019).

[14] P. Geri, S. El Yacoubi, and C. Goyet, "Forecast of sea surface acidification in the northwestern mediterranean sea," Journal of Computational Environmental Sciences, 2014. DOI: 10.1155/2014/201819. [Online]. Available: https://www.hindawi.com/journals/jces/2014/201819/ abs/ (visited on 09/09/2019).

[15] T. Midorikawa, H. Y. Inoue, M. Ishii, D. Sasano, N. Kosugi, G. Hashida, S.-i. Nakaoka, and T. Suzuki, "Decreasing $\mathrm{pH}$ trend estimated from 35-year time series of carbonate parameters in the pacific sector of the southern ocean in summer," Deep Sea Research Part I: Oceanographic Research Papers, vol. 61, pp. 131-139, Mar. 1, 2012, ISSN: 0967-0637. DOI: $10.1016 /$ j . dsr. 2011.12.003. [Online]. Available: http : / / www . sciencedirect . com / science / article / pii / S0967063711002354 (visited on 09/09/2019).

[16] W. S. Sarle, "Neural networks and statistical models," in Proceedings of the Nineteenth Annual SAS Users Group International Conference, Cary, North Carolina, Apr. 1994.

[17] NASA Goddard Space Flight Center, Ocean Ecology Laboratory, Ocean Biology Processing Group, "SuomiNPP/VIIRS level 3 mapped particulate inorganic carbon (2018 reprocessing) [dataset]," NASA OB.DAAC, 2018. DOI: data / 10 . 5067 / NPP / VIIRS / L3M / PIC / 2018 [Online]. Available: https://oceancolor.gsfc.nasa.gov/ data/10.5067/NPP/VIIRS/L3M/PIC/2018/.

[18] AIRS Science Team/Joao Teixeira, "AIRS/aqua 13 8-day $\mathrm{CO} 2$ in the free troposphere (AIRS-only) 2.5 degrees $\mathrm{x}$ 2 degrees (version 5) [dataset]," Goddard Earth Sciences and Information Services Center, 2009. DOI: 10. 5067/Aqua/AIRS/DATA334 [Online]. Available: https: //disc.gsfc.nasa.gov/datacollection/AIRX3C28_005. html.

[19] NASA Goddard Space Flight Center, Ocean Ecology Laboratory, Ocean Biology Processing Group, "SuomiNPP/VIIRS level 3 mapped particulate organic carbon (2018 reprocessing) [dataset]," NASA OB.DAAC, 2018. DOI: data / 10 . 5067/NPP/VIIRS / L3M / POC / 2018 [Online]. Available: https://oceancolor.gsfc.nasa.gov/ data/10.5067/NPP/VIIRS/L3M/POC/2018/.

[20] Y. LeCun, L. Bottou, G. B. Orr, and K.-R. Müller, "Efficient backprop," in Neural Networks: Tricks of the Trade, Berlin, Heidelberg: Springer-Verlag, Jan. 1, 1998, pp. 9-50, ISBN: 978-3-540-65311-0. 
[21] D. P. Kingma and J. Ba, "Adam: A method for stochastic optimization," presented at the 3rd International Conference for Learning Representations, San Diego, Dec. 22, 2014. arXiv: 1412.6980 [Online]. Available: http://arxiv.org/abs/1412.6980 (visited on 02/21/2020).

[22] S. Arlot and M. Lerasle, "Choice of $v$ for v-fold cross-validation in least-squares density estimation," The Journal of Machine Learning Research, vol. 17, no. 1, pp. 7256-7305, Jan. 1, 2016, ISSN: 1532-4435. 